\title{
Relación de la autorregulación infantil con sensibilidad materna y contexto familiar a los 12 y 30 meses de edad
}

\section{Relationship between children self-regulation, maternal sensitivity and fa- mily context at 12 and $\mathbf{3 0}$ months of age}

\author{
Alexandra Epstein, Catalina Pesce, Catalina Errázuriz, Isidora Gómez-Barris, Victoria Izquierdo, Chamarrita Farkas \\ Pontificia Universidad Católica de Chile, Chile
}

(Rec.: enero de 2018 - Acept.: abril de 2018)

Resumen

La autorregulación se refiere a la capacidad del niño/a para modificar su conducta según las demandas del entorno. Esta presenta una gran influencia en el desarrollo infantil, especialmente en el ámbito social. Desde una mirada evolutiva, la autorregulación, como característica temperamental, depende de factores tanto biológicos como contextuales, donde las figuras parentales juegan un rol determinante. En el siguiente estudio se analizó la relación entre la sensibilidad de la madre cuando el niño/a tenía 12 meses y la autorregulación de este tanto a los 12 como a los 30 meses de edad, para examinar si la sensibilidad materna predice la autorregulación infantil a los 30 meses. Además se incluyeron variables del contexto familiar, como tipo de familia, tipo de hijo/a y NSE. La muestra incluyó 72 diadas madre-hijo/a, con un diseño descriptivo, longitudinal, comparativo y correlacional. Se aplicó la Escala de Sensibilidad del Adulto (E.S.A.), un cuestionario sociodemográfico y los cuestionarios de temperamento IBQ-R-VSF y ECBQ-VSF. Los resultados muestran una relación significativa entre la autorregulación de los infantes a los 12 meses, con el tipo de hijo/a que son, y la interacción entre NSE y sensibilidad de las madres. A los 30 meses, en cambio, además de la autorregulación a los 12 meses, solo resulta ser un predictor significativo el tipo de hijo/a. Se discuten las implicancias de los resultados.

Palabras claves: Autorregulación, sensibilidad materna, nivel socioeconómico, tipo de familia, infancia temprana.

\begin{abstract}
Self-regulation refers to the capacity of a child to modify his or her behavior according to environmental demands. It strongly influences child's development, especially in the social sphere. From an evolutionary point of view, self-regulation, viewed as a temperamental characteristic, depends on biological and environmental factors, where the parental figures of the child play a crucial role. In the following study, an analysis of the relationship between mother's sensitivity when her child was 12 months old and the child's self-regulation at 12 months and 30 months of age was made. This was done to analyze if mother's sensitivity predicts child's self-regulation at 30 months of age. Also variables related to the family context were included, considering type of family, type of child and SES. The sample included 72 mother-child dyads, with a descriptive, longitudinal, comparative and correlational design. The Adult Sensitivity Scale, a socio-demographic questionnaire and the temperament questionnaires IBQ-R-VSF and ECBQ-VSF were used to obtain the data. The results show that there is a significant relationship between infants' self-regulation at 12 months, the type of child, and the interaction between SES and maternal sensitivity. At 30 months of age instead, additionally to the self-regulation at 12 months, only the type of child is a significant predictor. Further implications of these results are discussed.
\end{abstract}

Keywords: Self-regulation, maternal sensitivity, socioeconomic status, type of family, early infancy

Correspondencia: Chamarrita Farkas. Escuela de Psicología. Pontificia Universidad Católica de Chile. Av. Vicuña Mackenna 4860, Macul, Santiago. Correo electrónico: chfarkas@uc.cl

\footnotetext{
1 Nota autor

"Artículo de investigación que contó con la financiación otorgada por el Fondo Nacional de Desarrollo Científico y Tecnológico, FONDECYT, № 1110087 y 1160110"
} 


\section{Introducción}

\section{Autorregulación Infantil}

Aunque la autorregulación ha sido abordada desde distintos puntos de vista, en este estudio es entendida desde un punto de vista temperamental, como una disposición con la cual el niño/a nace, que le va a facilitar o dificultar la manifestación de la regulación frente a ciertas exigencias del contexto (Rothbart \& Derryberry, 1981). Entendiendo temperamento como la base constitucional o biológica de las diferencias individuales que afectan tanto en la reactividad como en la autorregulación de las emociones (Gartstein \& Rothbart, 2003), la autorregulación se refiere al proceso de atención consciente que puede servir para regular la reactividad (Posner \& Rothbart, 2000; Razza, Martin, \& Brooks-Gunn, 2011). Es una habilidad de alta complejidad que considera capacidades como cumplir solicitudes, comenzar y terminar una determinada tarea demandada, ser capaz de modular la intensidad de aquello que se realiza, y por último, para llevar a cabo conductas socialmente aprobadas en ausencia de ayuda externa (Kopp, 1982).

Parte de la autorregulación es el control con esfuerzo (Posner \& Rothbart, 2000), entendido como la habilidad de suprimir una respuesta dominante para ejecutar una respuesta menos dominante. Emerge entre los 6 y 12 meses de edad, para ir adquiriendo una importancia mayor hacia los dos años, siendo considerada como una competencia que se va desarrollando a lo largo del tiempo (González, Carranza, Fuentes, Galián \& Estévez, 2001). Hacia los 45 meses, el control con esfuerzo es estable y coherente tanto longitudinalmente como a través de una serie de tareas, siendo considerado como una característica temperamental del niño/a (Kochanska \& Knaack, 2003). El control con esfuerzo en niños/as preescolares se ha asociado con la función ejecutiva y la habilidad de demora de la respuesta, lo cual ha demostrado estar fuertemente vinculado al nivel de ingreso familiar, competencias sociales y una menor frecuencia de problemas de ajuste (Lengua et al., 2015). Además, predice una mayor conciencia en el niño/a a los 56 meses y menos problemas externalizados a los 73 meses (Kochanska \& Knaack, 2003). El control con esfuerzo y la autorregulación presentan diferencias individuales y son de gran relevancia para el desarrollo socioemocional infantil (Eisenberg, Hofer, Sulik, \& Spinrad, 2014).

Inicialmente la regulación infantil es co-regulada por la disposición del niño/a y por los padres, para luego transitar hacia una regulación interna, donde el niño/a interioriza y genera sus propios mecanismos de control (Ato, González \& Carranza, 2004). La autorregulación ha sido relacionada con la atención, motivación y persistencia e inhibición de conductas impulsivas (Caspi \& Silva, 1995; Magallón, Crespo-Eguilaz, Ecay, Poch-Olivé \& Narbona, 2009). Además, se relaciona con el desarrollo de la atención ejecutiva, fenómeno que tiene lugar durante toda la niñez, si bien la inmadurez durante el primer año de vida provoca que el niño/a requiera de reguladores externos tales como sus cuidadores (Gartstein \& Rothbart, 2003). Así, el desarrollo de la autorregulación en la infancia no solo depende de factores tales como la maduración del cerebro y los logros cognitivo-lingüísticos de los niños/as, sino de las influencias del contexto y la disponibilidad emocional de sus cuidadores, destacando aquí el importante papel que juegan los padres (González et al., 2001).
En el presente estudio se buscó investigar acerca de la autorregulación infantil y su relación con la sensibilidad materna. Específicamente, se buscaba analizar si la sensibilidad materna era un predictor de la autorregulación infantil tanto a los 12 como a los 30 meses, sustentado en que muchas de las actitudes y recursos internos de los niños/as (incluyendo la autorregulación y la autoevaluación) están fuertemente influenciados por las actitudes y comportamientos de los padres (Grolnick \& Ryan, 1989). Se consideraron ambas edades ya que al año de vida del niño/a ya manifiesta los inicios de regulación, pero es aún muy dependiente de su madre, mientras que a los 3 años ya tiene más independencia para manifestar sus propios comportamientos. Además, dentro del segundo y tercer año de vida los niños/as, estos ya han adoptado de cierta manera las normas familiares y sociales propias, lo cual queda evidenciado en muchos de sus comportamientos (Kopp, 1982).

\section{Aspectos Relacionados con la Autorregulación Infantil}

La autorregulación influye en diversos aspectos de la vida del niño/a, por tanto, si la sensibilidad materna resulta ser un predictor de la misma, ello tendría importantes implicancias en programas de intervención. Otro aspecto que pueden incidir en la autorregulación infantil desde el contexto familiar es la presencia/ausencia del padre y de hermanos, aspectos que fueron incluidos en este estudio. Finalmente, el nivel socioeconómico (NSE) familiar también ha demostrado ser una variable importante por su relación con la autorregulación del niño/a.

\section{Sensibilidad materna}

El papel de las madres en el desarrollo de la autorregulación de los niños/as es fundamental. El tiempo que ellas pasan con sus hijo/as, el tipo de control y respuestas que manifiestan, en especial, ante situaciones de estrés, influencian sin duda el comportamiento del niño/a (Esquivel, García, Montero \& Valencia, 2013). La sensibilidad de la madre se entiende como una habilidad para tomar conciencia de las señales del niño/a, interpretar lo que necesita y responder de forma apropiada y contingente a sus señales (Ainsworth, Blehar, Waters \& Wall, 1978). Se ha detectado en diversos estudios que padres sensibles y atentos tienden a generar relaciones seguras con sus hijos/as a los 12 meses y al contrario, padres insensibles tienen mayor riesgo de establecer vínculos inseguros (Ainsworth \& Bell, 1972; Santelices, M. P., Carvacho, C., Farkas, C., León, F., Galleguillos, F., \& Himmel, E. 2012). Además, la sensibilidad puede tener efectos en otras aristas del desarrollo del niño/a. Ainsworth y Bell (1972) evidencian que la interacción entre la madre y el hijo/a tiene una fuerte influencia en el desarrollo social del niño/a, donde una respuesta apropiada de la madre, tiende a acelerar el desarrollo de una comunicación clara y heterogénea y de ciertos aspectos del desarrollo de competencias sociales. Esto podría apuntar a una posible relación entre la sensibilidad de la madre y la autorregulación infantil, ya que madres sensibles a las señales de sus hijos/as sirven de apoyo a la autorregulación del niño/a y promueven el desarrollo social de este.

\section{Contexto familiar: Presencia de otros miembros de la familia}

Se cree que la presencia en el hogar de la figura del padre es importante porque según la teoría del apego, una de las características más influyentes en la conducta de base segura 
del niño/a es la manera en que los padres interactúan con ellos y la vivencia de la paternidad en sí (Ugarte \& Susana, 2004). A partir de los modelos de interacción cuidador-niño/a, la literatura expone que existen diferencias entre las relaciones padre-hijo/a y madre-hijo/a, debido a que la madre expresa más su sensibilidad en situaciones de cuidado, mientras que el padre la expresa en situaciones no estresantes, de juego, incrementando su participación en la vida del niño/a a partir del segundo año de vida (Bretherton, 2010; Cox, Tresch, Henderson \& Margand, 1992; Lamb, 1977), incentivando la exploración, las habilidades sociales y la autorregulación emocional (Cabrera, Tamis-LeMonda, Bradley, Hofferth \& Lamb, 2000).

La literatura que relaciona la autorregulación infantil y tipo de familia (monoparental versus biparental) es escasa. No obstante, se ha observado una relación significativa entre monoparentalidad y una menor autorregulación en niños/as preescolares (Bukres, McClelland \& \& Tracy, 2015), y una autorregulación emocional deficiente en adolescentes (Nobile, M., Bianchi, V., Bellina, M., Greco, A., Monzani, D., Tesei, A., \& Molteni, M. 2014).

Además, la presencia y las relaciones entre hermanos ha sido objeto de interés de los teóricos del desarrollo que estudian las relaciones afectivas en el contexto familiar (Ripoll, Carrillo \& Castro, 2009). Los y las adolescentes que perciben la relación con sus hermanos/as como positiva y cálida, presentan mayores niveles de ajuste psicológico (Oliva \& Arranz, 2005), y una mala calidad de dichas relaciones se asocia con predictores de conducta antisocial, especialmente en varones (Sobral, Romero \& Marzoa, 2000). No obstante, no existen estudios que contrasten la presencia (y número) de hermanos/as versus la situación de ser hijo/a único, con la autorregulación del niño/a, lo cual ha sido considerado en este estudio.

\section{Nivel socioeconómico}

EI NSE de la familia ha demostrado ser una importante variable tanto para las competencias de los padres como de los niños/as. Ciertos estudios muestran que madres con un mejor NSE y bagaje educacional, exhiben niveles más altos de sensibilidad hacia su hijo/a (Farkas, Carvacho, Galleguillos, Montoya, León, Santelices, \& Himmel, 2015; Santelices, Farkas, Montoya, Galleguillos, Carvacho, Fernández, \& Himmel, 2015). En el caso de la autorregulación infantil, un mayor NSE se relaciona de manera significativa con un mayor control con esfuerzo así como un incremento en la autorregulación de los niños/as, y sus expresiones como, por ejemplo, la función ejecutiva (Kochanska, \& Knaack, 2003; Lengua, Moran, Zalewski, Ruberry, Kiff, \& Thompson, 2015).

\section{El Presente Estudio}

El presente estudio tuvo como primer objetivo analizar la relación entre la sensibilidad materna y la autorregulación infantil, en un grupo de niños/as evaluados a los 12 meses, y luego a los 30 meses de vida. En este sentido, se esperaba poder describir la autorregulación infantil en ambas edades y analizar su relación con la sensibilidad materna (medida a los 12 meses de los niños/as) e indagar si esta era un predictor significativo de la autorregulación infantil a estas edades. El segundo objetivo fue analizar la relación entre la autorregulación infantil y el contexto familiar, considerando el tipo de familia (monoparental/biparental), la presencia y número de hermanos/as, tipo de hijo/a (hijo/a único, hijo/a individual con hermanos, mellizo o gemelo) y NSE, con el objetivo de aportar información sobre la posible relación de estos aspectos en la etapa de desarrollo en la que se encontraban. Además, cabe considerar que estos aspectos no han sido estudiados en población chilena. La hipótesis de partida, de acuerdo a los antecedentes presentados, apuntaba a que una mayor sensibilidad materna inicial se relacionaría con una mayor autorregulación infantil tanto a los 12 como a los 30 meses de edad en los niños/as evaluados. Además, se esperaba observar una mayor autorregulación en los niños/as de familias biparentales, que tuvieran hermanos y con un NSE alto.

\section{Método}

Se utilizó una metodología cuantitativa, con un diseño descriptivo, longitudinal, correlacional y comparativo. Se describió inicialmente la autorregulación infantil a los 12 y 30 meses de edad en un mismo grupo de niños/as, y se correlacionó con la sensibilidad materna medida a los 12 meses de los niños/as y con el número de hermanos/as. Luego se analizó posibles diferencias en autorregulación comparando la pertenencia a una familia biparental/monoparental, presencia/ausencia de hermanos/as, tipo de hijo/a y NSE.

\section{Participantes}

La muestra estuvo compuesta de 72 niños/as, $44.4 \%$ de sexo femenino $(n=32)$ y $55.6 \%$ de sexo masculino $(n=40)$, que asistían a salas cuna públicas y privadas, en la ciudad de Santiago. Al inicio del estudio, los niños/as tenían una edad ubicada en un rango entre 10 y 15 meses (media $=12.17, D . S .=1.35$ ). Al ser evaluados por segunda vez, los niños/as se ubicaron en un rango entre 27 y 33 meses (media $=29.92, D . S .=1.19$ ). El $52.8 \%$ de la muestra formaba parte de familias con un NSE bajo, el $23.6 \%$ pertenecía a familias con un NSE medio y el $23.6 \%$ estaba inserto en familias de un NSE alto. $22.5 \%$ de los casos eran familias monoparentales, y en el $54.2 \%$, el niño/a era hijo/a único. En los casos en que sí había hermanos/as, el rango iba de 1 a 3 hermanos/as (ver Tabla 1). Los criterios de inclusión en el estudio fueron que las madres vivieran con sus hijo/ as, que los niños/as asistieran a sala cuna, que sus edades estuvieran en el rango de 10-15 meses al inicio del estudio y que ni la madre ni su hijo/a presentaran alguna psicopatología relevante. 
Tabla 1. Características sociodemográficas de la muestra

\begin{tabular}{|c|c|c|c|c|c|}
\hline & $\mathrm{N}$ & Min. & Max. & Media & $\begin{array}{c}\text { Desviación } \\
\text { standard }\end{array}$ \\
\hline Edad del niño/a medición 1 & 72 & 10 & 15 & 12.17 & 1.353 \\
\hline Edad del niño/a medición 2 & 72 & 27 & 32 & 29.29 & 1.192 \\
\hline \multirow[t]{2}{*}{ Número de hermanos/as } & 72 & 0 & 3 & .57 & .728 \\
\hline & Frecuencia & Porcentaje & & & \\
\hline \multicolumn{6}{|l|}{ Sexo del niño } \\
\hline Masculino & 40 & $55.6 \%$ & & & \\
\hline Femenino & 32 & $44.4 \%$ & & & \\
\hline \multicolumn{6}{|l|}{ NSE } \\
\hline Bajo & 38 & $52.8 \%$ & & & \\
\hline Medio & 17 & $23.6 \%$ & & & \\
\hline Alto & 17 & $23.6 \%$ & & & \\
\hline \multicolumn{6}{|l|}{ Tipo de familia } \\
\hline Monoparental & 16 & $22.2 \%$ & & & \\
\hline Biparental & 55 & $76.4 \%$ & & & \\
\hline \multicolumn{6}{|l|}{ Tipo de hijo/a } \\
\hline Hijo/a único & 39 & $54.2 \%$ & & & \\
\hline Presencia de hermanos & 30 & $41.7 \%$ & & & \\
\hline Mellizo/gemelo & 3 & $4.2 \%$ & & & \\
\hline
\end{tabular}

\section{Instrumentos}

Cuestionario sociodemográfico. Este cuestionario fue desarrollado por el equipo de investigación y se aplicó para obtener información para caracterizar la muestra. Para este estudio se consideraron las variables sexo y edad de los niños/as, NSE, presencia y número de hermanos, y presencia/ausencia del padre en el hogar. Para la asignación del NSE se utilizó el índice ESOMAR (Adimark, 2000) que considera nivel educacional y ocupacional del principal sostenedor económico de la familia.

Escala de sensibilidad del adulto E.S.A. (Santelices et al., 2012). Este instrumento evalúa la sensibilidad en adultos significativos en interacción con niños/as de 6 a 36 meses, entendida como la capacidad de responder oportunamente a las señales y necesidades del niño/a, a modo de generar un efecto positivo, especialmente en el interés y cooperación en el juego (Santelices et al., 2012). Se filma una interacción de 5 minutos de juego libre entre el adulto y el niño/a, con un set standard de juguetes. Para la codificación, se utiliza una rúbrica de 19 indicadores, que dan cuenta de las conductas sensibles de la madre hacia el niño/a, en un rango de 1 a 3 puntos. A partir del promedio del puntaje asignado a cada indicador, se obtienen 3 escalas, producto del análisis factorial entre los ítems: (1) Responsividad, o capacidad de reconocimiento, interpretación atenta de respuestas y capacidad de reconocer las señales de un niño/a por parte de un adulto para responder así adecuadamente a sus demandas; (2) Interacción Lúdica, o capacidad del adulto para relacionarse con el niño/a, a través una interacción equilibrada en el juego y la participación de ambos de forma activa; (3) Sintonía, la cual refiere a un adulto con una actitud sensible y cálida frente a las necesidades y emociones del niño/a a través del lenguaje y de las acciones que realiza con él.
Finalmente, el instrumento arroja un puntaje global de sensibilidad en un rango de 1 a 3 puntos, y ubica al adulto en las categorías de sensibilidad "Baja", "Adecuada" y "Alta". Una sensibilidad baja apunta a un adulto con dificultades para percibir las señales del niño/a y responder adecuadamente a estas, una sensibilidad adecuada indica a un adulto que ocasionalmente puede captar las señales del niño/a y responder de manera apropiada a ellas, aunque esto no ocurre todo el tiempo. Una sensibilidad alta apunta a un adulto que en general capta las señales del niño/a, y que es capaz de responder a ellas de manera adecuada y oportuna, favoreciendo un clima positivo y reforzador con el niño/a. El instrumento tiene una buena confiabilidad (Coeficiente Alfa de Cronbach=0.930). Las filmaciones fueron codificadas por codificadores independientes previamente entrenados, basándose en 35 videos seleccionados al azar y cuya consistencia inter-jueces fue de 0.62 (Kappa de Cohen) (Santelices et al., 2012).

Cuestionarios de temperamento de Rothbart. Las escalas de temperamento diseñadas por Rothbart (Rothbart \& Derryberry, 1981), consisten en una evaluación a través del reporte de los padres respecto de diversas conductas del niño/a. Existen diferentes versiones según la edad del sujeto, pero para el presente estudio solo se utilizaron las que cubren la edad de los niños/as del estudio: IBQ-R-VSF y ECBQ-VSF.

El Infant Behavior Questionnaire Revised, Very Short Form (IBQ-R-VSF) (Putnam, Helbig, Gartstein, Rothbart \& Leerkes, 2014) es un cuestionario de reporte de conductas que responde el cuidador del niño/a entre los 3 y 12 meses de edad, donde los ítems del cuestionario evalúan la frecuencia de distintos tipos de comportamientos relativos al temperamento durante 
los últimos 7 días previos a la aplicación. La evaluación se realiza por medio de una escala Likert de 7 puntos, en la cual las frecuencias van desde "nunca" hasta "siempre". La versión abreviada, IBQ-R-VSF considera 36 ítems agrupados en 3 dimensiones: Extraversión, Afecto Negativo y Orientación/ regulación. La primera dimensión mide (mediante la agrupación de aproximación, reactividad vocal, alta intensidad del placer o goce, sonrisa y risa, nivel de actividad y sensibilidad perceptual) una dimensión de la personalidad semejante a la Extraversión. La segunda dimensión, Afecto Negativo, es análoga al rasgo de personalidad de Neuroticismo (caracterizado por tristeza, estrés ante las limitaciones, miedo y reactividad). Finalmente, la dimensión de Orientación/regulación, caracterizado por baja intensidad del placer o goce, ternura, duración de la orientación, sociabilidad, miedo social y enganche atencional, ha demostrado ser un predictor del control con esfuerzo (Putnam et al., 2014). El IBQ-R-VSF ha demostrado tener una adecuada consistencia (0.70-0.92 para extraversión; 0.72-0.88 para afecto negativo; 0.71-0.82 para regulación) (Putnam et al., 2014). En una muestra de 150 niños/as chilenos de características similares a la de este estudio, la confiabilidad medida con Alfa de Cronbach fue de .780 para la escala completa, y de .672 para la dimensión de extraversión, .800 para afecto negativo y .759 para regulación (Farkas, Santelices \& Himmel, 2011). Aunque este cuestionario está pensado para ser aplicado hasta los 12 meses, un estudio realizado con niños/as chilenos y norteamericanos de mayor edad, demostró que el cuestionario arrojaba una confiabilidad igual o mayor en los niños/as ubicados en el rango entre 13 y 15 meses, en comparación a los niños/as entre 10 y 12 meses (Farkas \& Vallotton, 2016).

El Early Childhood Behavior Questionnaire, Very Short Form (ECBQ-VSF) (Putnam, Gartstein, \& Rothbart, 2006) es muy similar al cuestionario ya descrito, pero está focalizado en niños/as entre 18 y 36 meses, y considera 36 ítems agrupados en las mismas 3 dimensiones. Las propiedades psicométricas del instrumento muestran una adecuada consistencia interna y una buena correlación entre cuidadores(as) primarios y secundarios (Putnam et al., 2006). En una muestra de 62 niños/ as chilenos de características similares a la de este estudio, la confiabilidad medida con Alfa de Cronbach fue de .719 para la escala completa, de .706 para la dimensión Extraversión, .756 para Afecto Negativo y .645 para Regulación (Farkas et al., 2011).

Los cuestionarios presentan una confiabilidad interna moderada para la mayoría de las escalas. Para este estudio solo se utilizaron los puntajes obtenidos en la dimensión de Regulación.

\section{Procedimiento}

El contacto con las madres se realizó a través de las salas cuna a las cuales asistían sus hijo/as, para lo cual se contó previamente con la autorización del centro educacional. Con anterioridad a las evaluaciones se pidió la firma de una carta de consentimiento informado a las madres, de modo de considerar los resguardos éticos pertinentes.

Al inicio del estudio se aplicó un cuestionario sociodemográfico a la familia. Se prosiguió completando el Cuestionario de temperamento de Rothbart (IBQ-R-VSF) por las madres, y la filmación de juego libre con el niño/a para su posterior codifi- cación con la Escala de Sensibilidad Adulto (E.S.A.). Las escalas fueron respondidas por las madres de los niños/as en los centros educativos a los cuales ellos asistían respectivamente. Las filmaciones de la interacción madre-bebé, que tenían una duración de 5 minutos de juego libre, se llevaron a cabo en el centro educativo, en una sala acondicionada para este fin. Uno de los principales requisitos para la realización de los videos, fue el cuidado de que la participación de los infantes siempre fuera voluntaria en términos de que este no se notara incómodo o irritable con la situación. Los niños/as volvieron a ser evaluados a los 30 meses de edad, momento en que se les pidió a las madres que respondieran el ECBQ-VSF.

\section{Análisis de Datos}

El análisis estadístico se realizó con el programa SPSS. En primera instancia, se realizaron análisis descriptivos de las variables del estudio; autorregulación de los niños/as a los 12 meses y 30 meses, y sensibilidad materna a los 12 meses. Luego, se analizó la relación entre autorregulación infantil a los 12 y 30 meses con la sensibilidad materna cuando el niño/a tenía 12 meses (considerando el puntaje total y en las 3 escalas, así como las categorías de sensibilidad alta, adecuada y baja) con análisis correlacionales (coeficientes de Pearson y Spearman). Posteriormente, se analizó la relación de la autorregulación infantil en los dos momentos evaluados con las variables del contexto familiar considerando el tipo de familia (monoparental, biparental), presencia/ausencia de hermanos, número de hermanos, tipo de hijo/a (hijo/a único, hijo/a individual con hermanos, mellizo/gemelo) y NSE, para lo cual se ocuparon análisis correlacionales y prueba t de Student. Finalmente, se realizaron análisis de regresión jerárquica a los 12 y 30 meses de edad de los niños/as, para analizar cuánta varianza de la autorregulación podían explicar las variables del estudio.

\section{Resultados}

\section{Análisis descriptivos de Autorregulación Infantil y Sensibilidad Materna}

En relación a los puntajes del niño/a en la escala de regulación, la media a los 12 meses de edad fue de 5.4 (D.S. = .64). En cambio, la media a los 30 meses fue de 5.0 (D.S. $=.59)$ (ver Tabla 2).

Tabla 2. Estadísticos descriptivos de la regulación infantil y sensibilidad materna

\begin{tabular}{|c|c|c|c|c|}
\hline & Min. & Max. & Media & D.S. \\
\hline Regulación 12m & 3.83 & 6.55 & 5.39 & 0.64 \\
\hline Regulación 30m & 3.31 & 6.31 & 4.96 & 0.59 \\
\hline $\begin{array}{l}\text { Escala de } \\
\text { responsividad }\end{array}$ & 1 & 3 & 1.98 & 0.55 \\
\hline $\begin{array}{l}\text { Escala de } \\
\text { interacción lúdica }\end{array}$ & 1 & 3 & 2.16 & 0.56 \\
\hline Escala de sintonía & 1.43 & 3 & 2.22 & 0.44 \\
\hline $\begin{array}{l}\text { Puntaje total } \\
\text { sensibilidad }\end{array}$ & 1.37 & 2.79 & 2.10 & 0.40 \\
\hline
\end{tabular}

Respecto a la sensibilidad de las madres cuando sus hijo/as tenían 12 meses de edad, un $23.5 \%$ de ellas presentaba una 
sensibilidad baja, mientras que el $76.5 \%$ presentaba una alta o adecuada sensibilidad (ver Figura 1). En la escala de sensibilidad total, ellas obtuvieron una media de $2.1(D . S .=.40)$, y una media de $1.98(D . S .=.55)$ en responsividad, $2.16(D . S .=.56)$ en interacción lúdica, y $2.22(D . S .=.43)$ en sintonía (ver Tabla 2).

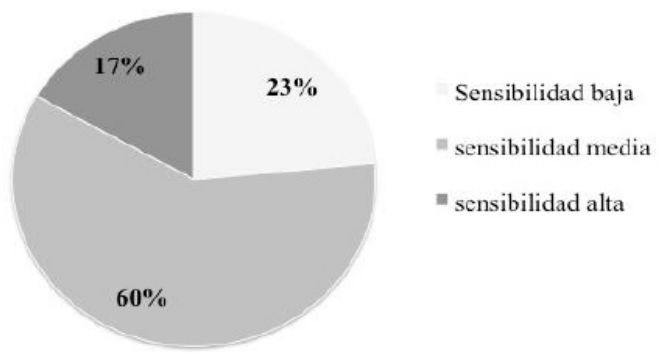

Figura 1: Distribución de las madres en categorías de sensibilidad a los 12 meses de edad del niño/a

\section{Relación entre Autorregulación Infantil y Sensibilidad Materna}

Cuando los niños/as tenían 12 meses de edad, se aprecia una correlación positiva moderada entre su regulación y la sensibilidad materna, en términos de que una mayor regulación de los niños/as se relacionaba tanto con un mayor puntaje total de sensibilidad de la madre $(r=.252, p=.034)$ así como un mayor puntaje en la escala de responsividad $(r=.234, p=$ .050) y una categoría de sensibilidad más alta $(r=.237, p=$ .046) (ver Tabla 3).

Cuando los niños/as tenían en cambio 30 meses de edad, no se apreciaron correlaciones entre la sensibilidad materna medida a los 12 meses y la regulación infantil, en ninguno de los aspectos evaluados.

Tabla 3: Análisis correlacionales entre regulación infantil y sensibilidad materna

\begin{tabular}{|c|c|c|c|c|c|c|c|}
\hline & & 1 & 2 & 3 & 4 & 5 & 6 \\
\hline $\begin{array}{l}\text { 1. Regulación } \\
12 \text { meses }\end{array}$ & $\begin{array}{l}\text { Pearson } \\
\text { Corr. Sig. }\end{array}$ & 1 & & & & & \\
\hline 2.Regulación & Pearson & $.414^{* *}$ & 1 & & & & \\
\hline 30 meses & Corr. Sig. & .000 & & & & & \\
\hline \multirow{2}{*}{$\begin{array}{l}\text { 3. Escala } \\
\text { Responsivi- } \\
\text { dad }\end{array}$} & Pearson & $.234^{*}$ & .112 & 1 & & & \\
\hline & Corr. Sig. & .050 & .353 & & & & \\
\hline \multirow{2}{*}{$\begin{array}{l}\text { 4. Escala } \\
\text { Interacción } \\
\text { Lúdica }\end{array}$} & Pearson & .153 & .106 & $.375^{* *}$ & 1 & & \\
\hline & Corr. Sig. & .204 & .381 & .001 & & & \\
\hline 5. Escala & Pearson & .201 & .118 & $.593^{* *}$ & $.499^{* *}$ & 1 & \\
\hline Sintonía & Corr. Sig. & .092 & .327 & .000 & .000 & & \\
\hline \multirow{2}{*}{$\begin{array}{l}\text { 6. Puntaje } \\
\text { total } \\
\text { sensibilidad }\end{array}$} & Pearson & $.252^{*}$ & .156 & $860^{* *}$ & $.634^{* *}$ & $.877^{1 * *}$ & 1 \\
\hline & Corr. Sig. & .034 & .193 & .000 & .000 & .000 & \\
\hline \multirow{2}{*}{$\begin{array}{l}\text { 7. Categoría } \\
\text { sensibilidad }\end{array}$} & $\begin{array}{l}\text { Spe- } \\
\text { arman }\end{array}$ & $.237^{*}$ & .028 & $.773^{* *}$ & $.651^{\text {*k }}$ & $.732^{2 * *}$ & $.896^{* *}$ \\
\hline & $\begin{array}{l}\text { Corr. } \\
\text { Sig. }\end{array}$ & .046 & .817 & .000 & .000 & .000 & .000 \\
\hline
\end{tabular}

Nota: $* * p<.001, * p<.05$
Relación entre autorregulación infantil a los 12 y 30 meses, con variables del contexto familiar

Los análisis correlacionales entre regulación infantil y cantidad de hermanos/as no mostraron resultados significativos (ver Tabla 4).

Los análisis comparativos realizados no muestran diferencias significativas en la regulación del niño/a a los 12 o 30 meses al considerar los tipos de familia (monoparental, biparental) o la presencia/ausencia de hermanos/as. En cambio sí se aprecian diferencias en regulación a los 12 meses según el tipo de hijo/a que el niño/a es $(F(2,69)=6.977, p=.002)$, donde los niños/as que son gemelos o mellizos obtienen puntuaciones más bajas en regulación que aquellos niños/as que son hijo/ as únicos ( $L S D=-1.297, p=.001)$, o hijo/as individuales con hermanos ( $L S D=-1.218, p=.001)$. $A$ los 30 meses de edad, esta diferencia desaparece. También solo en los 12 meses se aprecia una tendencia en relación a NSE $(F(2,69)=2.999, p=$ .056), donde los niños/as con NSE bajo obtienen significativamente puntajes más bajos en regulación que los niños/as con NSE alto ( $L S D=-0.430, p=.021)$ (ver Tabla 5).

Tabla 4. Análisis correlacionales entre regulación infantil y número de hermanos/as

\begin{tabular}{llll}
\hline & & 1 & 2 \\
\hline 1. Regulación 12 meses & $\begin{array}{l}\text { Pearson Corr. } \\
\text { Sig. }\end{array}$ & & \\
& 2. Regulación 30 meses & & \\
& Pearson Corr. & $.414^{* *}$ & 1 \\
& Sig. & .000 & \\
Número de hermanos/as & Pearson Corr. & -.141 & .144 \\
& Sig. & .239 & .227 \\
\hline
\end{tabular}

Nota: ** $\mathrm{p}<.01, * \mathrm{p}<.05$

Tabla 5. Análisis comparativos entre regulación infantil y variables del contexto familiar

Tipo de familia

\begin{tabular}{|c|c|c|c|c|}
\hline & $\begin{array}{l}\text { Monoparen- } \\
\text { tal1 }\end{array}$ & Biparental1 & $F(p)$ & \\
\hline Regulación 12 meses & $5.28(0.68)$ & $5.41(0.62)$ & $0.455(.522)$ & \\
\hline Regulación 30 meses & $4.85(0.76)$ & $4.98(0.53)$ & $0.576(.451)$ & \\
\hline \multicolumn{5}{|c|}{ Presencia/ausencia de hermanos/as } \\
\hline & Ausencia & Presencia & & \\
\hline Regulación 12 meses & $5.48(0.52)$ & $5.29(0.75)$ & $1.590(.211)$ & \\
\hline Regulación 30 meses & $4.89(0.62)$ & $5.04(0.55)$ & $1.217(.274)$ & \\
\hline \multicolumn{5}{|c|}{ Tipo de hijo/a } \\
\hline & $\begin{array}{l}\text { Hijo/a } \\
\text { único }\end{array}$ & $\begin{array}{c}\mathrm{Hijo/a} \\
\text { individual } \\
\text { con } \\
\text { hermanos }\end{array}$ & $\begin{array}{l}\text { Mellizo/ } \\
\text { gemelo }\end{array}$ & \\
\hline Regulación 12 meses & $5.48(0.52)$ & $5.40(0.69)$ & $4.18(0.32)$ & $6.608^{* *}(.002)$ \\
\hline \multirow[t]{3}{*}{ Regulación 30 meses } & $4.89(0.62)$ & $5.08(0.55)$ & $4.72(0.24)$ & $1.118(.333)$ \\
\hline & & NSE & & \\
\hline & Bajo & Medio & Alto & \\
\hline Regulación 12 meses & $5.29(0.67)$ & $5.31(0.53)$ & $5.72(0.60)$ & $2.999+(.056)$ \\
\hline Regulación 30 meses & $4.86(0.58)$ & $5.08(0.57)$ & $5.06(0.62)$ & $1.113(.338)$ \\
\hline
\end{tabular}

1 Media y DS. ** $p<.01, \uparrow p<.07$ 
Análisis de regresión jerárquica para la autorregulación infantil a los 12 y 30 meses

Primero para explicar la autorregulación del niño/a a los 12 meses, se realizaron diversos análisis de regresión jerárquica, considerando las variables que previamente resultaron significativas. El modelo que aquí se reporta es una síntesis de lo que resultó ser más significativo. Así, se consideró primero el NSE de la familia (Modelo 1), luego el puntaje total de sensibilidad materna (Modelo 2), luego la interacción entre NSE y sensibilidad materna (Modelo 3 ) y finalmente el tipo de hijo/a (Modelo 4). El NSE es un predictor significativo $(\beta=.257, \mathrm{t}=$ $2.213, p=.030)$, explicando un $6.6 \%$ de la varianza del constructo (ver Modelo 1, Tabla 6), pero luego su efecto deja de ser significativo por sí solo al ingresar el resto de las variables. La sensibilidad materna no es un predictor significativo una vez se ha controlado el NSE (ver Modelo 2 Tabla 6). En cambio, la interacción entre NSE y sensibilidad materna sí es un predictor significativo ( $\beta=.282, t=2.445, p=.017$ ), explicando un $8.0 \%$ de la varianza, y se mantiene significativo al incluirse la variable tipo de hijo/a. El tipo de hijo/a también es un predictor significativo $(\beta=-.243, t=-2.150, p=.035)$, explicando el $5.9 \%$ de la varianza (ver Modelo 4 Tabla 6 ). El modelo final que incluye la interacción de NSE y sensibilidad materna, y el tipo de hijo/a resulta significativo $(F(2,68)=5.456, p=.006)$ y explica un $13.8 \%$ de la varianza de la variable.

En síntesis, de acuerdo a estos análisis, la interacción de un mayor NSE con una sensibilidad materna más alta, y el ser hijo/a único o tener hermanos (pero no ser mellizo o gemelo), predicen de manera significativa la autorregulación de los niños/as de esta muestra a los 12 meses de edad.

Tabla 6. Regresión jerárquica de la autorregulación infantil a los 12 meses

\begin{tabular}{|c|c|c|c|c|c|c|c|c|c|c|c|c|}
\hline & \multicolumn{3}{|c|}{ Modelo 1} & \multicolumn{3}{|c|}{ Modelo 2} & \multicolumn{3}{|c|}{ Modelo 3} & \multicolumn{3}{|c|}{ Modelo 4} \\
\hline & B & SE B & $\beta$ & B & SE B & $\beta$ & B & SE B & $\beta$ & B & SE B & $\beta$ \\
\hline Constante & 5.047 & .172 & & 4.649 & .410 & & 5.093 & .143 & & 5.250 & .157 & \\
\hline NSE & .200 & .090 & $.257 *$ & .129 & .112 & .166 & & & & & & \\
\hline Sensibilidad total & & & & .248 & .232 & .154 & & & & & & \\
\hline NSE*sensibilidad & & & & & & & .078 & .032 & $.282^{*}$ & .073 & .031 & $.263^{*}$ \\
\hline Tipo de hijo/a & & & & & & & & & & .269 & .125 & $.243^{*}$ \\
\hline R2 & & .066 & & & .082 & & & .080 & & & .138 & \\
\hline $\mathrm{F}$ & & 4.897* & & & $3.026 \dagger$ & & & $5.976 *$ & & & $5.456 * *$ & \\
\hline
\end{tabular}

Nota: $+p<0.07, * p<0.05, * * p<0.01$

Finalmente, se realizó un análisis de regresión jerárquica de la autorregulación del niño/a a los 30 meses, ingresando primero la autorregulación del niño/a a los 12 meses (Modelo 1), luego, la autorregulación del niño/a con el NSE y la sensibilidad materna por separados (Modelo 2), a continuación, la autorregulación del niño/a con la interacción entre NSE y sensibilidad (Modelo 3) y finalmente, la autorregulación del niño/a, NSE, sensibilidad materna, y el tipo de hijo/a (Modelo 4). El modelo final es significativo $(F(4,66)=4.893, p=.002)$ y explica el $25.6 \%$ de la varianza de la variable. El análisis muestra que el mejor predictor de la autorregulación del niño/a a los 30 meses, es la autorregulación a los 12 meses $(\beta=.419, \mathrm{t}=$ $3.832, \mathrm{p}<.000)$, explicando por sí sola un $16.9 \%$ de la varianza de la autorregulación a los 30 meses, y se mantiene significativa una vez se han incorporado las demás variables (ver Tabla 7). En esta ocasión la sensibilidad materna y NSE no son significativas ni por sí solas ni en interacción (ver Modelos 2 y 3, Tabla 7). El tipo de hijo/a también es significativo ( $\beta=.294$, $\mathrm{t}=2.438, \mathrm{p}=.018)$, agregando un $7.8 \%$ de varianza adicional (ver Modelo 4 Tabla 7).

En síntesis, de acuerdo a estos análisis una mayor autorregulación del niño/a a los 12 meses, y el tener hermanos (pero no ser mellizo o gemelo), predicen de manera significativa la autorregulación de los niños/as de esta muestra a los 30 meses de edad.

Tabla 7. Regresión jerárquica de la autorregulación infantil a los 30 meses

\begin{tabular}{|c|c|c|c|c|c|c|c|c|c|c|c|c|}
\hline & \multicolumn{3}{|c|}{ Modelo 1} & \multicolumn{3}{|c|}{ Modelo 2} & \multicolumn{3}{|c|}{ Modelo 3} & \multicolumn{3}{|c|}{ Modelo 4} \\
\hline & $\mathrm{B}$ & SE B & $\beta$ & B & SE B & $\beta$ & $\mathrm{B}$ & SE B & $\beta$ & $\mathrm{B}$ & SE B & $\beta$ \\
\hline Constante & 2.902 & .543 & & 2.819 & .608 & & 2.927 & .548 & & 2.032 & .693 & \\
\hline Autorregul. 12m. & .383 & .100 & $.419 * *$ & .369 & .106 & $.403^{* *}$ & .368 & .105 & $.402^{* *}$ & .448 & .117 & $.478^{* *}$ \\
\hline NSE & & & & .011 & .098 & .016 & & & & -.023 & .090 & -.032 \\
\hline Sensibilidad total & & & & .066 & .203 & .045 & & & & .181 & .180 & .124 \\
\hline NSE*sensibilidad & & & & & & & .015 & .029 & .059 & & & \\
\hline Tipo de hijo/a & & & & & & & & & & .304 & .125 & $.294^{*}$ \\
\hline $\mathrm{R} 2$ & & .175 & & & .178 & & & .179 & & & .256 & \\
\hline $\mathrm{F}$ & & $14.683^{* *}$ & & & $4.848 * *$ & & & $7.399 * *$ & & & $4.893^{* *}$ & \\
\hline
\end{tabular}

Nota: ${ }^{*} p<0.05, * * p<0.01$ 


\section{Discusión}

El primer objetivo de este estudio fue analizar la relación entre la autorregulación del niño/a tanto a los 12 como a los 30 meses, y la sensibilidad de la madre medida a los 12 meses de edad del niño/a. Se esperaba una relación significativa entre estas variables, en términos de que los niños/as más autorregulados a ambas edades tendrían madres con una mayor sensibilidad. Los análisis realizados muestran que la sensibilidad materna medida al año de edad del niño/a es una variable relevante para la autorregulación infantil en esta etapa, pero no aporta mayor influencia en su autorregulación a los 30 meses.

En relación a lo anterior, se puede pensar que estos resultados pueden deberse a que los niños/as a los 12 meses se encuentran mayormente en interacción con su madre, por lo que requieren en mayor medida de su sensibilidad y cuidado permanente para regular tanto sus emociones como otros ámbitos de su vida. Los infantes de un año de edad requieren de una adecuada sensibilidad del cuidador para que entienda y responda a sus demandas (Esquivel et al., 2013). Esto se complementa con lo que plantea Gartstein y Rothbart (2003), en relación a que efectivamente a los 12 meses de edad se tiene aún una inmadurez en el sistema atencional, y que por esto se requiere de una mayor y constante participación de reguladores externos, lo cual hace pensar que a esta edad la relación con la sensibilidad materna es más significativa. Al mismo tiempo, muestra que la sensibilidad materna es relevante en la autorregulación del niño/a cuando este es menor, y ya que la autorregulación a los 12 meses resulta ser el mejor predictor de la autorregulación del niño/a a los 30 meses, las variables significativas a los 12 meses, como lo es la sensibilidad materna, deben ser consideradas en programas de intervención que fortalezcan las competencias parentales durante el primer año de vida del infante.

Al año, el infante es completamente dependiente de sus cuidadores, por lo cual necesita del cuidado y cercanía de su madre para lograr regularse de mejor manera. Sin embargo, con casi 3 años, es un niño/a que tal como menciona Kopp (1982), ya ha adoptado normas familiares y sociales, lo cual le permite ser más consciente y activo en una gran variedad de situaciones en las cuales ya no cuenta con la presencia de su madre en todo momento. Además, muchos niños/as a esta edad ya asisten una mayor cantidad de horas a sus respectivos jardines infantiles, y por ende, comparten un buen porcentaje diario con otros pequeños de su misma edad así como con otros cuidadores (como el personal educativo), los cuales podrían cobrar más relevancia en el posterior desarrollo de la autorregulación del niño/a.

Un segundo objetivo de este estudio era analizar la relación entre la autorregulación del niño/a tanto a los 12 como a los 30 meses, con variables del contexto familiar, donde se consideró la presencia del padre, presencia y número de hermanos, tipo de hijo/a y NSE. La presencia o ausencia del padre del hogar no resultó significativa para la autorregulación ni a los 12 ni a los 30 meses de edad del niño/a. Ello podría interpretarse como que no es relevante la presencia de la figura paterna para la socialización de este rasgo temperamental, o por el contrario, que la influencia del padre no depende de si este vive o no con el niño/a, lo cual es positivo considerando el número creciente de familias monoparentales en nuestro país. Futuros estudios deberían considerar el peso de variables que indiquen más bien el tipo de relación padre-hijo/a, calidad de la misma y frecuencia de contacto.

En cuanto a la presencia y número de hermanos/as, estos no resultaron ser significativos para la autorregulación del niño/a, pero sí el tipo de hijo/a que el niño/a era, es decir, si era hijo/a único, si tenía hermanos pero era un hijo/a individual, o si tenía un mellizo o gemelo. Los niños/as que tenían un hermano mellizo o gemelo tenían una autorregulación menor a los 12 y 30 meses, incluso al controlarse las demás variables del estudio, mientras que a los 30 meses los niños/as que tenían hermanos y que al mismo tiempo no eran mellizos/gemelos, obtuvieron una mayor autorregulación. Estos resultados deben ser tomados con cautela debido al escaso número de niños/as de la muestra que se hallaban en la condición de ser mellizos/gemelos, pero abre al mismo tiempo preguntas interesantes sobre las dinámicas entre hermanos, las cuales parecieran ser más relevantes que el número de hermanos, y cómo estas afectan la capacidad del niño/a de autorregularse. Estudios futuros podrían considerar un mayor número de hermanos mellizos/gemelos, el lugar del niño/a entre sus hermanos, dinámicas según sexo, etc.

Los resultados del estudio avalan además la hipótesis de que la presencia de hermanos ayudaría a la autorregulación del niño/a. Esto muestra que, además de la figura materna, el contexto cercano al niño/a, representado en sus hermanos, va cobrando relevancia en la socialización de su manera de comportarse y regularse. Estudios futuros podrían considerar niños/as de mayor edad, para analizar si esta influencia se mantiene o aumenta, así como el rol de otras personas cercanas al niño/a como pares y personal educativo, especialmente en aquellos casos en que los niños/as entran tempranamente al sistema preescolar, y donde un "buen comportamiento", resultado de una adecuada capacidad de autorregulación, es valorado en la cultura chilena.

En relación al NSE, se esperaba observar que esta variable tuviera una importante influencia, como lo han demostrado una serie de estudios en población chilena en otros aspectos del desarrollo del niño/a, así como en competencias parentales. En este estudio el NSE tuvo un efecto menor, solo apreciándose una menor autorregulación en los niños/as de NSE en comparación a los niños/as de NSE alto a los 12 meses, pero luego ello desaparecía a los 30 meses. Asimismo, en los análisis de regresión al considerar las demás variables, el NSE era significativo solo a los 12 meses y solo al interactuar con la sensibilidad materna. Podría hipotetizarse entonces, que el NSE no afecta directamente la autorregulación del niño/a sino que más bien influye en las competencias del adulto, las que afectan más directamente al niño/a como lo es el caso de la sensibilidad materna, relación que ya ha sido descrita previamente en estudios chilenos (Farkas et al., 2015; Santelices et al., 2015).

Otra hipótesis para entender estos resultados, es que en la cultura chilena la autorregulación en los niños/as desde pequeños es muy valorada y estimulada, observándose que niños/as chilenos, por ejemplo a los 12 meses, ya obtienen puntajes más altos en autorregulación en comparación a niños/as norteamericanos (Farkas \& Vallotton, 2016). Otros estudios en preescolares chilenos han demostrado que los niños/as demuestran niveles más altos de autocontrol en tareas de demora de la gratificación, en comparación a normas 
norteamericanas (Barata, 2011; Yoshikawa, Barata, Rolla, Da Silva, Ayoub, Arbour \& Snow, 2008). Ello puede entenderse desde el concepto de distancia de poder, entendido como la manera en que cada cultura lidia con las diferencias de estatus y jerarquías de sociales. Las personas pertenecientes a una cultura con una baja distancia de poder (como Estados Unidos) valoran las relaciones simétricas y horizontales, y una distribución igualitaria del poder, mientras que personas de culturas con mayor distancia de poder (como Chile) tienden a aceptar mejor relaciones asimétricas y verticales, con una distribución no equitativa del poder, y donde las recompensas y sanciones dependen en ocasiones más del rango, estatus, edad o incluso sexo de la persona, que de su desempeño (Hofstede, Hofstede \& Minkov, 2010). Así, podría plantearse que en la sociedad chilena la autorregulación en los niños/as desde pequeños podría verse fomentada, sin grandes diferencias, según grupo socioeconómico.

Cabe mencionar que el mejor predictor de la autorregulación a los 30 meses fue el mismo constructo a los 12 meses. Ello confirma que pese al cambio de instrumento, estamos midiendo un mismo constructo, el cual es estable en el tiempo, lo cual es coherente con la literatura. Además, pone el acento en la relevancia de las intervenciones tempranas en aquellos aspectos que promueven una adecuada autorregulación, para que este efecto se mantenga en el tiempo.

Como posibles limitaciones del estudio cabe mencionar el pequeño tamaño muestral, el que todos los niños/as evaluados asistían a salas cuna, y que todos vivían en la ciudad de Santiago, lo cual limita la posibilidad de generalizar de los resultados y por tanto, estos resultados deben tomarse con cautela. Al mismo tiempo estos datos, aunque iniciales, permiten fundamentar la relevancia de las competencias maternas como la sensibilidad, en el desarrollo de aspectos del niño/a al año de edad, etapa en la cual deberían centrarse las intervenciones preventivas. Finalmente, se hace necesaria la inclusión en futuros estudios de otros factores familiares que promuevan o que sean factores potenciales de riesgo para la autorregulación del niño/a, generando así una mayor comprensión de su desarrollo y permitiendo el diseño de intervenciones específicas a cada contexto particular en la que se encuentran los niños/as.

\section{Referencias}

Adimark (2000). El nivel socioeconómico ESOMAR; Manual de aplicación. Recuperado de www.microweb.cl/ idm/documentos/ESOMAR.pdf

Ainsworth, M., \& Bell, S. M. (1972). Mother-infant interaction and the development of competence. New York, U.S.A.: Grant Foundation.

Ainsworth, M., Blehar, M., Waters, E. \& Wall, S. (1978). Patterns of attachment: A psychological study of the strange situation. New Jersey, U.S.A.: Lawrence Erlbaum Associates.

Ato, E., González, M., \& Carranza, J. A. (2004). Aspectos evolutivos de la autorregulación emocional en la infancia. Anales de Psicología, 20(1), 69-79.

Barata, C. (2011). Executive Function Skills in Chilean Preschool Children (Doctoral Dissertation). Retrieved from ProQuest Information \& Learning, 2012. AAI3486021. Harvard Graduate School of Education, Cambridge, MA.
Bretherton, I. (2010). Fathers in attachment theory and research: A review. Early Child Development and Care, 180(1/2), 9-23. doi:10.1080/03004430903414661

Bukres, A., McClelland, M. M., \& Tracy, A. (2015). Relations between parental marital status, residential mobility, and children's academic achievement and self-regulation in kindergarten. Oregon State University. Retrieved from http://ir.library.oregonstate.edu/ xmlui/bitstream/handle/1957/57420/Bukres\%20 URAP\%20Poster.pdf?sequence $=3$

Cabrera, N., Tamis-LeMonda, C., Bradley, R., Hofferth, S. \& Lamb, M. (2000). Fatherhood in the Twenty-First Century. Child Development, 71(1), 127-136. doi:10.1111/1467-8624.00126

Caspi, A., \& Silva, P. A. (1995). Temperamental qualities at age three predict personality traits in young adulthood: Longitudinal evidence from a birth cohort. Child Development, 66(2), 486-498. doi:10.2307/1131592

Cox, M., Tresch, M., Henderson, V. K. \& Margand, N. (1992). Prediction of infant-father and infant-mother attachment. Developmental Psychology, 28(3), 474483. doi:10.1037/0012-1649.28.3.474

Eisenberg, N., Hofer, C., Sulik, M. J., \& Spinrad, T. L. (2014). Self-regulation, effortful control, and their socioemotional correlates. In J. J. Gross (Ed.), Handbook of emotion regulation (pp. 157-172). New York: Guilford Press.

Esquivel, M., García, B., Montero, M., \& Valencia, A. (2013). Regulación materna y esfuerzo de control emocional en niños/as pequeños. International Journal of Psychological Research, 6(1), 30-40.

Farkas, C., Carvacho, C., Galleguillos, F., Montoya, F., León, F., Santelices, M. P., \& Himmel, E. (2015). Estudio comparativo de la sensibilidad entre madres y personal educativo en interacción con niños/as y niñas de un año de edad. Perfiles Educativos, 37(148), 16-33.

Farkas, C., Santelices, M. P., \& Himmel, E. (2011). Análisis desde una perspectiva evolutiva y cultural del uso de la comunicación gestual en infantes y pre-escolares, en la expresión y comprensión de los estados internos y su impacto en el desarrollo socio-emocional de los niños(as). Proyecto Fondecyt № 1110087. Santiago, Chile: Pontificia Universidad Católica de Chile, Escuela de Psicología.

Farkas, C., \& Vallotton, C. (2016). Differences in infant temperament in Chile and the US. Infant Behavior \& Development, 44, 208-218. doi:10.1016/j.infbeh.2016.07.005

Gartstein, M. A., \& Rothbart, M. K. (2003). Studying infant temperament via the Revised Infant Behavior Questionnaire. Infant Behavior and Development, 22(1), 64-86. doi:10.1016/S0163-6383(02)00169-8

González, C., Carranza, J. A., Fuentes, L. J., Galián, M. D., \& Estévez, A. F. (2001). Mecanismos atencionales y desarrollo de la autorregulación en la infancia. Anales de Psicología, 17(2), 275-286.

Grolnick, W. S., \& Ryan, R. M. (1989). Parent styles associated with children's self-regulation and competence in school. Journal of Educational Psychology, 81(2), 143. doi:10.1037/0022-0663.81.2.143

Hofstede, G., Hofstede, G. J., \& Minkov, M. (2010). Cultures and organizations: Software of the mind, 3rd edition. USA: McGraw-Hill.

Kochanska, G., \& Knaack, A. (2003). Effortful control as a personality characteristic of young children: An- 
tecedents, correlates, and consequences. Journal of Personality, 71(6), 1087-1112. doi:10.1111/14676494.7106008

Kopp, C. B. (1982). Antecedents of self-regulation: A developmental perspective. Developmental Psychology, 18(2), 199. doi:10.1037/0012-1649.18.2.199

Lamb, E. (1977). The development of mother-infant and father-infant attachments in the second year of life. Developmental Psychology, 13(6), 637-648. doi:10.1037/0012-1649.13.6.637

Lengua, L. J., Moran, L., Zalewski, M., Ruberry, E., Kiff, C., \& Thompson, S. (2015). Relations of growth in effortful control to family income, cumulative risk, and adjustment in preschool-age children. Journal of Abnormal Child Psychology, 43(4), 705-720. doi:10.1007/s10802-014-9941-2

Magallón, S., Crespo-Eguilaz, N., Ecay, M., Poch-Olivé, M. L., \& Narbona, J. (2009). Estilo comportamental al inicio del segundo año de vida: Estudio retrospectivo en escolares afectados de trastorno por déficit de atención e hiperactividad. Anales de Pediatría, 70(6), 562-569.

Nobile, M., Bianchi, V., Bellina, M., Greco, A., Monzani, D., Tesei, A., \& Molteni, M. (2014). EPA-1746-The role of family structure and of tryptophan hydroxylase 2 (TPH2) on the stability of deficient emotional self-regulation symptoms throughout adolescence. European Psychiatry, 29 (Suplement 1), 1. doi:10.1016/S09249338(14)78877-7

Oliva, A., \& Arranz, E. (2005). Sibling relationships during adolescence. European Journal of Developmental Psychology, 2, 253-270. doi:10.1080/17405620544000002

Posner, M., \& Rothbart, M. K. (2000). Developing mechanisms of self-regulation. Development and Psychopathology, 12(3), 427-441. doi:10.1017/ S0954579400003096

Putnam, S., Gartstein, M., \& Rothbart, M. (2006). Measurement of fine-grained aspects of toddler temperament: The Early Childhood Behavior Questionnaire. Infant Behavior and Development, 29(3), 386-401. doi:10.1016/j.infbeh.2006.01.004

Putnam, S. P., Helbig, A. L., Gartstein, M. A., Rothbart, M. K., \& Leerkes, E. (2014). Development and assessment of short and very short forms of the Infant Behavior Questionnaire-Revised. Journal of Personality Assessment, 96(4), 445-458.

Razza, R. A., Martin, A., \& Brooks-Gunn, J. (2011). Anger and children's socioemotional development: Can parenting elicit a positive side to a negative emotion? Journal of Child and Family Studies, 21(5) 845-856. doi:10.1007/s10826-011-9545-1

Ripoll, K., Carrillo, S., \& Castro, J. (2009). Relación entre hermanos y ajuste psicológico en adolescentes: los efectos de la calidad de la relación padres-hijo/ as. Avances en Psicología Latinoamericana, 27(1), 125-142.

Rothbart, M. K., \& Derryberry, D. (1981). Development of individual differences in temperament. Advances in Developmental Psychology, 1(1), 37-86.

Santelices, M. P., Carvacho, C., Farkas, C., León, F., Galleguillos, F., \& Himmel, E. (2012). Medición de la sensibilidad del adulto con niños/as de 6 a 36 meses de edad: Construcción y análisis preliminares de la Escala de Sensibilidad del Adulto, ESA. Terapia
Psicológica, 30(3), 19-29.

Santelices, M. P., Farkas, C., Montoya, M. F., Galleguillos, F., Carvacho, C., Fernández, A., \& Himmel, E. (2015). Factores predictivos de sensibilidad materna en infancia temprana. Psicoperspectivas, 14(1), 66-76. doi:10.5027/psicoperspectivas-Vol14-Issue1-fulltext-441

Sobral, J., Romero, E., \& Marzoa, J. (2000). Personalidad y conducta antisocial: Amplificadores individuales de los efectos contextuales. Psicothema, 12(4), 661670.

Ugarte, A., \& Susana, A. (2004). Conducta de base segura con el padre y representaciones de apego en niños/as preescolares. Tesis para optar al grado de licenciatura en Psicología, Universidad Católica del Perú, Lima, Perú.

Yoshikawa, H., Barata, M. C, Rolla, A., Da Silva, C. D., Ayoub, C, Arbour, M., \& Snow, C. E. (2008). Un Buen Comienzo, una iniciativa para mejorar la educación preescolar en Chile: Resultados del primer año de implementación. Report to the UNICEF office, Santiago, Chile. 